\title{
Expression of integrins and GDF9 mRNAs is associated with ovarian follicle size and donor puberty status in pigs 1 )
}

\author{
PAWEŁ ANTOSIK*, MICHAL JESETA**, WIES ŁAWA KRANC***, ADRIAN CHACHUŁA***, \\ ARTUR BRYJA***, JOANNA BUDNA ${ }^{* * * *}$, SYLWIA CIESIÓŁKA ${ }^{* * * *}$, \\ KATARZYNA WOJTANOWICZ-MARKIEWICZ*, ****, DOROTA BUKOWSKA*, \\ KLAUS-PETER BRÜSSOW*, MAŁGORZATA BRUSKA***, MICHAŁ NOWICKI****, \\ PAVLINA HULINSKA*****, MARIE MACHATKOVA*****, BARTOSZ KEMPISTY***, ****

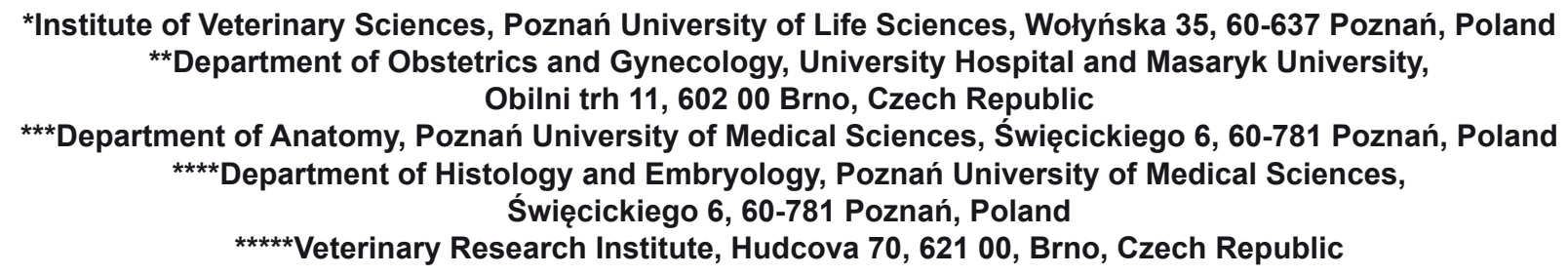

Antosik P., Jeseta M., Kranc W., Chachuła A., Bryja A., Budna J., Ciesiółka S., Wojtanowicz-Markiewicz K., Bukowska D., Brüssow K. P., Bruska M., Nowicki M., Hulinska P., Machatkova M., Kempisty B.

Expression of integrins and GDF9 mRNAs is associated with ovarian follicle size and donor puberty status in pigs

\section{Summary}

Ovarian folliculogenesis and oogenesis has a significant impact on embryo growth and development in preimplantation stages. Although both processes are widely understood in several species of mammals, including pigs, the factors influencing the proper maturation capability of oocytes, as well as the developmental competence of the surrounding somatic granulosa cells (GCs) and cumulus cells (CCs), are still not entirely known. This study aimed to investigate the expression of growth differentiation factor 9 (GDF9) and integrins (ITGB1, ITGB2, ITGB3 and ITGB4) in porcine oocytes isolated from follicles of various size and donors characterized by different puberty status. The relative abundance of GDF9, ITGB1, ITGB2, ITGB3, and ITGB4 mRNAs in porcine oocytes isolated from medium follicles of cycling sows (MFCS), small follicles of juvenile gilts (SFJG), and small follicles of cycling sows (SFCS) was assessed by an RT-qPCR assay. It was found an increased expression of GDF9 in oocytes isolated from the small follicles of juvenile gilts as compared to the other two groups $(\mathrm{P}<0.001)$. A significant down-regulation of ITGB1 and ITGB2 oocyte mRNAs collected from medium follicles of cycling sows was observed $(\mathrm{P}<0.05$ and $\mathrm{P}<0.001$, respectively). The ITGB3 $\mathrm{mRNA}$ expression was significantly decreased in oocytes isolated from small follicles of juvenile gilts $(P<0.001)$, whereas a lower expression of ITGB4 in oocytes from both medium follicles (cycling sows) and small follicles (juvenile gilts) was observed. In conclusion, GDF9 may be recognized as the main factor regulating follicle growth at early stages of folliculogenesis. The expression of ITGBs is significantly regulated by the puberty status of donor pigs, and different follicular sizes may play a subordinate role in integrin expression during in vivo follicle development in pigs.

Keywords: GDF9, IGFB, oocyte, pig

The proper development of cumulus-oocyte complexes (COCs) is orchestrated by the normal course of folliculogenesis and oogenesis. It is well accepted

Publication of this article was made possible by grant number 2014/13/D/ NZ9/04798 "SONATA" from the Polish National Centre of Science LD14104 COST-CZ MSMT, MH CZ - DRO (FNBr, 65269705) and funds from the Faculty of Medicine MU granted to the junior researcher Michal Jeseta. that folliculogenesis and oogenesis are associated with many morphological, molecular, and metabolic changes within growing ovarian follicles and COCs $(8,11,12$, $19,20,27,28,30)$. These modifications involve the secretion of multiple factors, including growth factors and hormones that regulate synchronous nuclear 
chromatin reorganization within oocytes as well as the remodeling of the cytoplasmic organelles of oocytes. It has also been seen that simultaneous changes within follicles significantly influence the growth and development of oocytes. Mammalian oocytes are surrounded by a complex of somatic cumulus cells (CCs) that may form either a compact or expanded architecture, which is dependent on the maturational stage of the oocyte (3, $12,22)$. The pivotal role of CCs in the oocyte-somatic cell "dialog" is well recognized in several species of mammals, although the molecular mechanisms/biochemical pathways involved in this process require further investigation. Additionally, it has been observed that proper communication between these two cell populations significantly influences the maturational competence and fertilizability of oocytes (9).

There are several available data indicating the role of growth factors, such as growth/differentiation factor 9 (GDF9), in the ability of mammalian oocytes, including those of domestic pigs, to reach full maturational capability $(10,14)$. Moreover, the expression of GDF9 during folliculogenesis, oogenesis, and early embryo growth has been well determined $(21,25,26)$. The role of GDF9, a member of the TGFB protein superfamily, in mammalian ovarian folliculogenesis in mammals was first defined by Dong et al. They used GDF9-deficient mice and observed complete infertility as a result of a block in follicle formation beyond the primary 1-layer stage of follicle growth. Moreover, oocytes underwent normal development, but oocyte-cumulus differentiation was significantly disturbed. In conclusion, they found that GDF9 was the first oocyte-derived growth factor responsible for the growth and development of somatic cells (7). A more recent study by Incerti et al. identified GDF9 as the main factor expressed in female gametes from the primary 1-layer follicle stage (15). Additionally, using in situ hybridization, McGrath et al. observed that GDF9 is expressed in oocytes at all stages of follicle growth except primordial follicular development (23). However, the influence of the follicular size or donor puberty status on the regulation of GDF9 expression in pigs is not entirely known.

Oocyte fertilizability is achieved only by fully mature oocytes, since only MII stage gametes with the proper amount of stored mRNAs and proteins are available for fertilization by a single spermatozoon. The extrinsic and intrinsic factors influencing the formation of a fully fertilizable female gamete are well defined, but the molecular pathways or markers that induce this process require further exploration. Our recent study indicated that zona pellucida proteins (ZP1, ZP2, ZP3, and $\mathrm{ZP} 4$ ) and integrins (ITGB) belong to the main factors influencing the fertilizability of porcine oocytes in vitro $(17,18)$. However, the expression of selected integrins, such as ITGB1, ITGB2, ITGB3, and ITGB4, in porcine oocytes, relative to the ovarian follicular size or puberty status of donor animals, was investigated in this study for the first time.
Since follicular size or donor puberty status may regulate the expression of selected genes influencing the maturational capability or fertilizability of porcine oocytes, this study aimed to investigate the expression profile of GDF9, ITGB1, ITGB2, ITGB3, and ITGB4 mRNAs in oocytes isolated from follicles of different sizes collected from various aged donors.

\section{Material and methods}

Animals. Crossbred animals (Landrace X Czech Large White pigs) were used as oocyte donors. The animals were divided into two groups: juvenile gilts that had not yet had piglets and adult sows that had already had piglets. Ovaries from the adult and juvenile cyclic sows were obtained from a local abattoir and transported to the laboratory within two hours at $31-33^{\circ} \mathrm{C}$. The ovaries of each donor were evaluated in terms of corpus luteum morphology and follicle population. For oocyte collection, only ovaries from cyclic sows from the late luteal to the early follicular phase (before dominant follicle progression) were used as described in Hulinska et al. (13). Only oocytes in the GV stage were included in this study.

Sample collection. Oocytes were recovered from follicles located on the ovaries. They were separated from medium follicles with greater meiotic competence (3-5 $\mathrm{mm}$ ) and small follicles with lesser meiotic competence $(<3 \mathrm{~mm})$ by aspiration and cutting of the ovarian cortex, respectively. Oocytes from larger follicles ( $\geq 5 \mathrm{~mm}$ ) were not included in this study. All healthy COCs with dark, evenly granulated ooplasm and at least two cumulus layers were used for experiments. After isolation, oocytes were manually denuded from CCs with a narrow pipette into TCM-199 medium containing 1\% NBCS and $0.1 \%$ hyaluronidase (w/v), and transported into $1 \mathrm{ml}$ TRI reagent (T9424 Sigma) in a $1.5 \mathrm{ml}$ eppendorf-type tube (for RT-qPCR analyses) or into PBS with $0.4 \%$ BSA (for CGs staining).

RNA extraction from oocytes. Total RNA was extracted from the samples with TRI Reagent (Sigma, St Louis, MO, USA) and RNeasy MinElute cleanup Kit (Qiagen, Hilden, Germany). The amount of total mRNA was determined from optical density at $260 \mathrm{~nm}$, and the RNA purity was estimated using the 260/280 $\mathrm{nm}$ absorption ratio (higher than 1.8) (NanoDrop spectrophotometer, Thermo Scientific, ALAB, Poland). The RNA integrity and quality were evaluated using a Bioanalyzer 2100 (Agilent Technologies, Inc., Santa Clara, CA, USA). The resulting RNA integrity numbers (RINs) were between 8.5 and 10 with an average of 9.2 (Agilent Technologies, Inc., Santa Clara, CA, USA). RNA in each sample was diluted to a concentration of $100 \mathrm{ng} / \mu \mathrm{l}$ with an OD260/280 ratio of $1.8 / 2.0$.

RT-qPCR analysis of GDF9, ITGB1, ITGB2, ITGB3, and ITGB expression in porcine oocytes. Reverse transcription was performed using AMV reverse transcriptase (Promega) with Oligo dT (PE Biosystems, Warrington, UK) as primers at a temperature of $42^{\circ} \mathrm{C}$ for $60 \mathrm{~min}$ (thermocycler UNO II, Biometra). The primers used were designed with the Primer 3 software (Whitehead Institute for Biomedical Research, Cambridge, MA) (Tab. 2). The primers were purchased from the Laboratory of DNA Sequencing and Oligonucleotide Synthesis (Institute of Biochemistry and Biophysics, Polish Academy of Sciences, Warsaw). RT-qPCR was performed using a Lightcycler 2.0 instrument (Roche Life Science, Mannheim, Germany) with software version 4.05. 
With the above-mentioned primers, the SYBR green detection system was used. Each of $20 \mu 1$ reaction mixtures contained $4 \mu 1$ template cDNA (standard or control), $0.5 \mu \mathrm{M}$ of every gene-specific primer, and a previously determined optimal $\mathrm{MgCl}_{2}$ concentration (3.5 $\mu \mathrm{M}$ for one reaction). LightCycler FastStart DNA Master SYBR Green I mix (Roche Diagnostic, Mannheim, Germany) was used. The real-time PCR program included a 10 min denaturation step to activate the Taq DNA Polymerase, followed by a three-step amplification program: denaturation, annealing, and extension. The specificity of the reaction products was checked by the determination of melting points $\left(0.1^{\circ} \mathrm{C} / \mathrm{s}\right.$ transition rate $)$. The results of $\mathrm{mRNA}$ expression encoding ZP1 and ZP4 genes were quantified in relation to two housekeeping genes, HPRT and ACTB.

Statistical analysis. A one-way ANOVA, followed by Tukey's post-hoc test, was used to compare the results of the real-time quantification of the proliferation index. The experiments were carried out in a minimum of three replicates. The differences were considered to be significant at $* P<0.05$, $* * P<0.01$, and $* * * P<0.001$ for the quantitative analyses of mRNA expression. The software program GraphPad Prism version 4.0 (GraphPad Software, San Diego, CA) was used for statistical calculations.

\section{Results and discussion}

Using RT-qPCR assays, we analyzed the expression of ITGB1, ITGB2, ITGB3, ITGB4, and GDF9 mRNAs in porcine oocytes isolated from medium follicles of cycling sows (MFCS), small follicles of juvenile gilts (SFJG), and small follicles of cycling sows (SFCS). Lower expression of ITGB1 mRNA was observed in oocytes collected from MFCS $(P<0.05)$ (Fig. 1A). Similarly, the relative abundance of ITGB2 mRNA was decreased in MFCS as compared to SFJG and SFCS $(P<0.001)$ (Fig. 1B). The analysis of the ITGB3 transcript revealed a signifi-

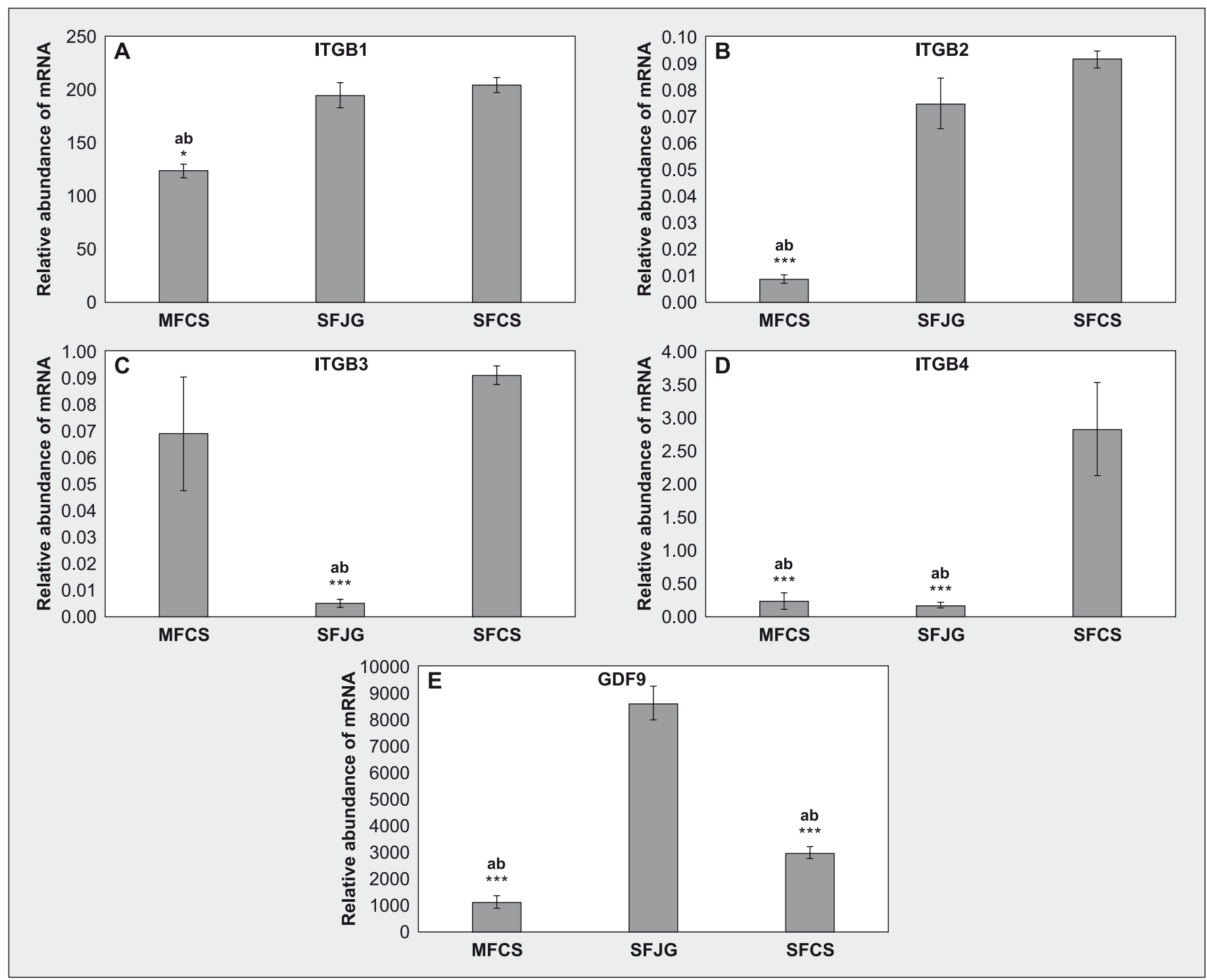

Fig. 1. Relative abundance of ITGB1, ITGB2, ITGB3, ITGB4, and GDF9 transcripts in porcine oocytes isolated from follicles of different sizes

Explanations: Porcine oocytes were isolated from medium follicles of cycling sows (MFCS), small follicles of juvenile gilts (SFJG), and small follicles of cycling sows (SFCS), and immediately used to isolate RNA, which was reverse-transcribed into cDNA. The relative abundance of ITGB1 (Fig. 1A), ITGB2 (Fig. 1B), ITGB3 (Fig. 1C), ITGB4 (Fig. 1D), and GDF9 (Fig. 1E) mRNAs was evaluated by RQ-PCR analysis before and after IVC. The results are presented as the mean \pm SEM with the level of significance shown as $* \mathrm{P}<0.05, * * \mathrm{P}<0.01$, and $* * * \mathrm{P}<0.001$. Different superscripts indicate significant differences (ab). 
cant down-regulation in oocytes collected from SFJG $(P<0.001)$ (Fig. 1C). The expression of ITGB4 mRNA was decreased in MFCS and SFJG as compared to SFCS $(P<0.001$ for both groups, respectively) (Fig. 1D). We found that GDF9 was up-regulated in oocytes isolated from the SFJG group as compared to MFCS and SFCS $(P<0.001$ for both groups, respectively) (Fig. 1E).

The goal of this study was to investigate the expression of GDF9, ITGB1, ITGB2, ITGB3, and ITGB4 in porcine oocytes collected from follicles of different sizes originating from donors at various puberty stages.

Our recent study focused on the expression analysis of TGFB genes including TGFB1, TGFB2, TGFB3, and GDF9 in porcine oocytes before and after in vitro maturation (IVM), as well as in gametes collected from follicles of various sizes (16). We found an increased expression of TGFB1 and TGFB2 in oocytes isolated from larger size follicles compared to medium and small ones, whereas TGFB3 and GDF9 expression was unchanged. Additionally, the relative abundance of TGFB1 and GDF9 mRNA was higher in oocytes after IVM compared to those before IVM. It has been suggested that TGFB expression is significantly regulated by oocyte maturational capability and the size of follicles. Moreover, GDF9 may be recognized as a factor that highly regulates the developmental capacity of porcine oocytes. Indeed, Caixeta et al. (6) used a follicle size model of Bos indicus to investigate the molecular markers of oocyte developmental competence. They examined the expression profile of H1Foo, H2A, H3A, GHR, GDF9, BMP15, and OOSP1 in oocytes, and of FSHR, EGFR, GHR, PTX3, and IGFII in surrounding somatic CCs. The COCs were matured, fertilized, and developed in vitro, whereas denuded oocytes or CCs were used for subsequent RT-qPCR assays. They observed an increased blastocyst rate from oocytes isolated from follicles of a larger size. Moreover, only the H2A transcript had a significantly higher expression in oocytes isolated from follicles of greater size, whereas in CCs this tendency was found in the expression of FSHR, EGFR, and GHR mRNA. It is suggested that oocytes and CCs differ significantly with regard to their target gene expression profiles.

The developmental competence of gametes and surrounding $\mathrm{CCs}$ is regulated by the activation of different molecular mechanisms. The role of GDF9 as a protein crucial for the regulation of folliculogenesis, oogenesis, as well as oocyte-somatic cell crosstalk, was widely presented. Additionally Myllymaa et al. (24) used recombinant bone morphogenic protein (BMP) type II receptor inhibitor (BMPR2ecd-Fc) to down-regulate both GDF9 and BMP15 expression in vitro. The recombinant inhibitor was injected intraperitoneally into neonatal mice, and early folliculogenesis during the postnatal days 4-12 was evaluated. They observed a significantly decreased mitogenesis of granulosa cells (GCs) and secondary and tertiary follicle numbers, whereas the size of oocytes was increased. It may be postulated that BMPR2ecd-Fc is a potent factor that regulates the proper course of folliculogenesis and modulates follicle growth in vivo. Hence,
GDF9 and BMP15 are involved in the regulation of folliculogenesis and may be recognized as oocyte-derived factors influencing ovarian follicle morphogenesis.

As there is a large population of follicles of different sizes covering the ovarian surface, as well as a very strict compaction between follicular somatic cells and oocytes, the gene expression profile specific for a separate cell population is of high interest. The gene expression pattern specific for GCs, CCs, or oocytes was recently studied by Bonnet et al. (2). They used laser capture microdissection (LCM) to distinguish an expression profile specific for follicular cells and oocytes in relation to sheep folliculogenesis. Their experiments included the evaluation of oocyte gene expression profiles specific for SOHLH2, MAEL, MATER, VASA, GDF9, BMP15, and folliclerelated genes - KL, GATA4, and AMH. Moreover, the Affymetrix microarray assay for the analysis of GCs and oocyte-specific transcriptomic profile was performed in this study. They found 1050 different mRNAs specific for GCs, and 759 were highly expressed in oocytes. They concluded that both LCM and microarray analyses may be used to distinguish gene expression profiles between denuded oocytes and follicular somatic cells. Similarly, it may be suggested that the oocyte and the surrounding follicular cells reflect differential gene expression profiles, although these cell populations are strictly compact.

Our current study demonstrated up-regulation of GDF9 expression in oocytes isolated from small follicles of juvenile gilts as compared to the other groups of sows. We propose that GDF9 is significantly associated with the proper course of early folliculogenesis and is the main factor involved in follicle growth during differentiation.

The integrins (ITGBs) belong to a family of leukocyte cell adhesion molecules and are mainly involved in processes of cell adhesion and cellular recognition during induction of specific immune response mechanisms. These membrane-specific glycoproteins are also recognized as factors involved in the inflammatory reaction. In addition, they are expressed during carcinogenesis. Several types of ITGBs are molecular markers of cancer cell growth, differentiation, and cancer metastasis. Because of their adhesion properties, ITGBs have recently been used as the markers of fertilization during gamete recognition and fusion. Moreover, our recent study clearly demonstrated the possible function of ITGBs as the markers of uterine receptivity in preimplantation periods in pigs, as well as during endometritispyometra complex disease in domestic bitches $(4,5)$. We also investigated the expression of zona pellucida proteins (ZP3) and integrins-beta 2 (ITGB2) in porcine oocytes after a single and double exposure to a brilliant cresyl blue (BCB) competence test. We found that ITGB2 mRNA was increased in porcine oocytes after a single $\mathrm{BCB}$ test as compared to both double staining and control oocytes before or after IVM. Additionally, significant changes in the nucleocytoplasmic shuttling of ITGB2 were also shown. In conclusion, we demonstrated that a single or double exposure to a BCB test significantly 
influenced the expression of both ZP3 and ITGB2, as well as their nucleocytoplasmic distribution.

ITGB2 may also be recognized as a maturational capability marker for porcine oocytes in in vitro culture (IVC). Antosik et al. (1) investigated the association between ovarian follicular size and the expression of fertilizability markers in porcine oocytes. In their experiments, RT-qPCR and Western-blot assays were used to analyze the ZP1, ZP2, ZP3, ZP4, ITGB1, and ITGB2 mRNAs, and ZP3 and ITGB2 proteins, respectively. They found an increased expression of ZP1, ZP3, ITGB1, and ITGB2 $\mathrm{mRNA}$ in porcine oocytes isolated from large and medium follicles as compared to small follicles. Western-blot analysis revealed an up-regulation of the ZP3 protein in oocytes collected from large and medium follicles as compared to small follicles. They concluded that follicular size significantly influenced the level of fertilizability markers mRNA and protein expression in pubertal gilts. There are few available data indicating an association between the expression profiles of ITGBs and ovarian follicular size in mammals. However, there are reports by Santos et al. (29), who investigated the early ovarian regression in the neotropical freshwater teleost curimatã-pacu (Prochilodus argenteus). They focused on follicular apoptosis and the basement membrane structure, as well as on ITGB1, collagen type IV, and caspase 3 protein staining. They reported on the expression of the above-mentioned proteins in the follicular layer of the postovulatory follicle and concluded that IGTB1 and collagen type IV may be recognized as molecular markers of ovarian follicular cell survival factors. The results of the current study clearly demonstrate a significant up-regulation of ITGB1, ITGB2, ITGB3, and ITGB4 in oocytes isolated from small follicles of cycling sows.

In conclusion, we propose that ovarian follicular size plays a subordinate role in the regulation of ITGBs expression in pigs. Moreover, the expression of ITGB3 and ITGB4 is significantly up-regulated by the donor puberty status, since significantly increased mRNA levels were observed in cycling sows.

\section{References}

1. Antosik P., Kempisty B., Bukowska D., Jackowska M., Wlodarczyk R., Budna J., Brussow K. P., Lianeri M., Jagodzinski P. P., Jaskowski J. M.: Follicular size is associated with the levels of transcripts and proteins of selected molecules responsible for the fertilization ability of oocytes of puberal gilts. J. Reprod. Dev. 2009, 55, 588-593.

2. Bonnet A., Bevilacqua C., Benne F., Bodin L., Cotinot C., Liaubet L., Sancristobal M., Sarry J., Terenina E., Martin P., Tosser-Klopp G., MandonPepin B.: Transcriptome profiling of sheep granulosa cells and oocytes during early follicular development obtained by laser capture microdissection. BMC Genomics 2011, 12, 417

3. Buccione R., Schroeder A. C., Eppig J. J.: Interactions between somatic cells and germ cells throughout mammalian oogenesis. Biol. Reprod. 1990, 43, 543-547.

4. Bukowska D., Kempisty B., Jackowska M., Wozna M., Antosik P., Piotrowska H. Jaskowski J. M.: Analysis of integrins and vascular endothelial growth factor isoforms mRNA expression in the canine uterus during perimplantation period. Pol. J. Vet. Sci. 2011, 14, 253-258.

5. Bukowska D., Kempisty B., Zawierucha P., Jopek K., Piotrowska H., Antosik P., Ciesiolka S., Wozna M., Brussow K. P., Jaskowski J. M.: Microarray analysis of inflammatory response-related gene expression in the uteri of dogs with pyometra. J. Biol. Regul. Homeost. Agents. 2014, 28, 637-648.

6. Caixeta E. S., Ripamonte P., Franco M. M., Junior J. B., Dode M. A.: Effect of follicle size on mRNA expression in cumulus cells and oocytes of Bos indicus: an approach to identify marker genes for developmental competence. Reprod. Fertil. Dev. 2009, 21, 655-664.
7. Dong J., Albertini D. F., Nishimori K., Kumar T. R., Lu N., Matzuk M. M.: Growth differentiation factor-9 is required during early ovarian folliculogenesis. Nature 1996, 383, 531-535.

8. Dunlop C. E., Anderson R. A.: The regulation and assessment of follicular growth. Scand. J. Clin. Lab. Invest. Suppl. 2014, 244, 13-7; discussion 17.

9. Eppig J. J.: Intercommunication between mammalian oocytes and companion somatic cells. BioEssays news rev. 1991, 13, 569-574.

10. Eppig J. J.: Oocyte control of ovarian follicular development and function in mammals. Reproduction 2001, 122, 829-838.

11. Eppig J. J., Wigglesworth K., Pendola F. L.: The mammalian oocyte orchestrates the rate of ovarian follicular development. Proc. Natl. Acad. Sci. USA 2002, 99 , 2890-2894.

12. Gilchrist R. B., Ritter L. J., Armstrong D. T.: Oocyte-somatic cell interactions during follicle development in mammals. Anim. Reprod. Sci. 2004, 82-83, 431-446.

13. Hulinska P., Martecikova S., Jeseta M., Machatkova M.: Efficiency of in vitro fertilization is influenced by the meiotic competence of porcine oocytes and time of their maturation. Anim. Reprod. Sci. 2011, 124, 112-117.

14. Hussein T. S., Thompson J. G., Gilchrist R. B.: Oocyte-secreted factors enhance oocyte developmental competence. Dev. Biol. 2006, 296, 514-521.

15. Incerti B., Dong J., Borsani G., Matzuk M. M.: Structure of the mouse growth/ differentiation factor 9 gene. Biochim. Biophys. Acta 1994, 1222, 125-128.

16. Jackowska M., Kempisty B., Wozna M., Piotrowska H., Antosik P., Zawierucha P., Bukowska D., Nowicki M., Jaskowski J. M., Brussow K. P.: Differential expression of GDF9, TGFB1, TGFB2 and TGFB3 in porcine oocytes isolated from follicles of different size before and after culture in vitro. Acta Vet. Hung. 2013, 61, 99-115.

17. Kempisty B., Jackowska M., Piotrowska H., Antosik P., Wozna M., Bukowska D., Brussow K. P., Jaskowski J. M.: Zona pellucida glycoprotein 3 (pZP3) and integrin beta2 (ITGB2) mRNA and protein expression in porcine oocytes after single and double exposure to brilliant cresyl blue test. Theriogenology 2011, $75,1525-1535$.

18. Kempisty B., Piotrowska H., Bukowska D., Wozna M., Ciesiolka S., WojtanowiczMarkiewicz K., Wlodarczyk R., Jopek K., Jeseta M., Bruska M., Nowicki M., Jaskowski J. M., Brussow K. P., Zabel M.: Expression and cellular distribution of zona pellucida glycoproteins in canine oocytes before and after in vitro maturation. Zygote 2015, 23, 863-873.

19. Kimura N., Hoshino Y., Totsukawa K., Sato E.: Cellular and molecular events during oocyte maturation in mammals: molecules of cumulus-oocyte complex matrix and signalling pathways regulating meiotic progression. Soc. Reprod. Fertil. Suppl. 2007, 63, 327-342.

20. Krisher R. L.: The effect of oocyte quality on development. J. Anim. Sci. 2004, 82 E-Suppl, E14-23.

21. Li Y., Li R. Q., Ou S. B., Zhang N. F., Ren L., Wei L. N., Zhang Q. X., Yang D. Z.: Increased GDF9 and BMP15 mRNA levels in cumulus granulosa cells correlate with oocyte maturation, fertilization, and embryo quality in humans. Reprod. Biol. Endocrinol. 2014, 12, 81.

22. Mattioli M., Galeati G., Seren E.: Effect of follicle somatic cells during pig oocyte maturation on egg penetrability and male pronucleus formation. Gamete Res. 1988, 20, 177-183.

23. McGrath S. A., Esquela A. F., Lee S. J.: Oocyte-specific expression of growth/ differentiation factor-9. Mol. Endocrinol. 1995, 9, 131-136.

24. Myllymaa S., Pasternack A., Mottershead D. G., Poutanen M., Pulkki M. M., Pelliniemi L. J., Ritvos O., Laitinen M. P.: Inhibition of oocyte growth factors in vivo modulates ovarian folliculogenesis in neonatal and immature mice. Reproduction 2010, 139, 587-598

25. Orisaka M., Jiang J. Y., Orisaka S., Kotsuji F., Tsang B. K.: Growth differentiation factor 9 promotes rat preantral follicle growth by up-regulating follicular androgen biosynthesis. Endocrinology 2009, 150, 2740-2748.

26. Paulini F., Melo E. O.: The role of oocyte-secreted factors GDF9 and BMP15 in follicular development and oogenesis. Reprod. Domest. Anim. 2011, 46, 354-361.

27. Piotrowska H., Kempisty B., Sosinska P., Ciesiółka S., Bukowska D., Antosik P. Rybska M., Brussow K. P., Nowicki M., Zabel M.: The role of TGF superfamily gene expression in the regulation of folliculogenesis and oogenesis in mammals: a review. Vet. Med. 2013, 58, 505-515.

28. Sanchez F., Smitz J.: Molecular control of oogenesis. Biochim. Biophys. Acta 2012, 1822, 1896-1912

29. Santos H. B., Sato Y., Moro L., Bazzoli N., Rizzo E.: Relationship among follicular apoptosis, integrin betal and collagen type IV during early ovarian regression in the teleost Prochilodus argenteus after induced spawning. Cell Tissue Res. 2008, 332, 159-170.

30. Sun Q. Y., Lai L., Bonk A., Prather R. S., Schatten H.: Cytoplasmic changes in relation to nuclear maturation and early embryo developmental potential of porcine oocytes: effects of gonadotropins, cumulus cells, follicular size, and protein synthesis inhibition. Mol. Reprod. Dev. 2001, 59, 192-198.

Corresponding author: Bartosz Kempisty PhD, Święcickiego 6, 60-781 Poznań, Poland; e-mail: bkempisty@ump.edu.pl 